\title{
Factors Influencing Efficacy of Complete Decongestive Treatment in Patients with Breast Cancer-Linked Arm Lymphoedema
}

\author{
Dragana Bojinović-Rodić, Samra Pjanić,' Tamara Popović,, 2 Tatjana \\ Nožica-Radulović1, 2
}

\section{Abstract}

Background/Aim: The most recommended form of lymphoedema therapy is complete decongestive treatment (CDT). Efficacy of CDT in patients with arm lymph-oedema related to malignant breast tumour has reported in many studies, but the predictive factors of outcome of this therapy have not been yet sufficiently investi-gated. The purpose of this research was to identify predictive factors of efficacy of CDT in patients with breast cancer-linked arm lymphoedema throughout the inten-sive phase of therapy.

Methods: The prospective study included 51 patients with breast cancer-linked arm lymphoedema who were subjected to a 3-week program of CDT. Patients' clini-cal and demographic features, breast cancer treatment characteristics, lymphoede-ma and CDT characteristics were collected and assessed for their prognostic value. The influence of certain predictors on the degree of lymphoedema reduction was evaluated by multivariate linear regression analysis.

Results: Mean age was $58.1 \pm 8.0$ (95 \% CI: 55.8 - 60.3), median of BMI was 28.4 $\mathrm{kg} / \mathrm{m}^{2}$ (95 \% CI: $\left.27.2-29.6\right)$. The average duration of lymphoedema was $36.5 \pm 43.9$ months (95 \% CI: 24.1 - 48.8). The mean size of lymphoedema before CDT was 6.99 $\pm 5.36 \%$, and the mean degree of lymphoedema reduction was $63.7 \pm 28.6 \%$. The mean compliance to bandages was $217.5 \pm 97.8$ hours (95 \% CI: $190.0-245.0$ ) and $7(13.7 \%)$ patients had a history of erysipelas of the ipsilateral arm. When observ-ing each individual predictor, statistically most significant contribution showed the size of lymphoedema before the therapy $(p<0.001)$, then history of erysipelas $(p<0.01)$, and patients' age $(p<0.05)$.

Conclusion: Size of lymphoedema before treatment is the most crucial prognostic factor of the efficacy of CDT in the patients with breast cancer-linked arm lymph-oedema. The present study also identified history of erysipelas and patients age as independent predictors of the CDT efficacy.

Key words: Breast cancer; Lymphoedema; Physical therapy modalities; Compression bandages; Drainage; Treatment outcome.

\section{Introduction}

Breast cancer-linked arm lymphoedema is ab-normal accumulation of interstitial fluid due to mechanical failure of the lymphatic system of the upper limb, usually because of the breast cancer
(1) Institute for Physical Medicine and Rehabilitation "Dr Miroslav Zotović", Banja Luka, the Republic of Srpska, Bosnia and Herzegovina.

(2) Faculty of Medicine, University of Banja Luka, Banja Luka, the Republic of Srpska, Bosnia and Herzegovina.

Correspondence:

DRAGANA BOJINOVIĆ-RODIĆ

E: dbojinovic@yahoo.com

M: +38765 422999

\section{ARTICLE INFO}

Received: 30 January 2021

Revision received: 25 May 2021 Accepted: 26 May 2021

Copyright $\odot 2021$ Bojinović-Rodić et al. This is an open access article distributed under the Creative Commons Attribution License (CC BY), which permits unrestricted use, distribution, and reproduction in any medium, provided the original work is properly cited. This article should be cited as follows: Bojinović-Rodić D, Pjanić S, Popović T, Nožica-Radulović T. Factors influencing efficacy of complete decongestive treatment in patients with breast cancer-linked arm lymphoedema. Scr Med 2021 Jun;52(2):132-7. 
after sentinel lymph node biopsy is less than 10 $\% .^{1-4}$ The overall goal of lymphoedema treat-ment is to reduce swelling, mobilise congestive interstitial fluid, reduce connective and fat tissue proliferation, control symptoms, and minimise the consequences. ${ }^{5}$

The most effective and most common form of lymphoedema therapy is complete decongestive treatment (CDT), which represents the gold standard in the conservative treatment. ${ }^{6-9}$ Efficacy of CDT in patients with arm lymphoedema related to malignant breast tumour has reported in many studies, ${ }^{10-14}$ but the predictive factors of outcome of this therapy have not been yet sufficiently in-vestigated. The purpose of this research was to identify independent predictive factors of effica-cy of CDT in patients with breast cancer-linked arm lymphoedema throughout the intensive phase of therapy.

\section{Methods}

\section{Study design}

This prospective study was carried out at the In-stitute for Physical Medicine and Rehabilitation "Dr Miroslav Zotović" in Banja Luka, and included patients with arm lymphoedema who underwent malignant breast tumour surgical procedure. The research was permitted by the Ethics Committee of the facility.

\section{Participants}

The inclusion criteria for the study were: unilateral axillary dissection, clinically verified lymphoede-ma (difference in circumference between affected and healthy arm was larger than $2 \mathrm{~cm}$ at minimum 1 measurement level), more than 3 months from the breast cancer surgery and radiotherapy and patient-signed informed consent form, with prior knowledge of the trial purpose.

The elimination criteria were: metastatic breast disease, clinically verified acute erysipelas; un-treated and poorly controlled hypertension, heart failure, deep venous thrombosis and anticoagulant therapy, shoulder and upper limb damage caused by neurological, orthopaedic or rheumatic diseas-es diagnosed prior to breast cancer surgery, diag-nosed and medically treated psychiatric disorders, liver cirrhosis and nephrotic syndrome. For each patients following data were collected and assessed for their prognostic value: clinical and de-mographic features (age, body mass index-BMI, co-morbidity), breast cancer treatment characteristics (time from surgery, type of breast surgery, number of lymph nodes removed and involved, therapy before and/or after surgery), lymphoedema char-acteristics (duration of lymphoedema, time until lymphoedema onset, size of lymphoedema, report-ing pain and other symptoms in the arm, history of erysipelas) and CDT characteristics (compliance to bandages).

\section{Intervention}

Patients were taken to a 3-week program of CDT, once a day, 5 days a week. The CDT protocol con-sisted of manual lymphatic drainage (MLD), short-stretch multilayer compression bandages (Rosidal ${ }^{\circledR}$ K Lymphset, Lohmann \& Rauscher, Vienna, Austria) and exercises provided by therapists. Exercises were performed with compression bandages as an essential part of the decongestive phase of lymph-oedema therapy. The exercises consisted of: exer-cises of diaphragmatic breathing, remedial exercis-es, flexibility (stretching) exercises and resistance (weight-lifting) exercises of the affected arm.

\section{Lymphoedema size}

Lymphoedema was assessed by the arm circum-ference. It was measured at 7 symmetrical levels (metacarpophalangeal joints, radial styloid process, at $10,20,30$ and $40 \mathrm{~cm}$ from the radial styloid pro-cess and over olecranon) of the affected and contra-lateral arm. The lymphoedema size was expressed as the ratio of the total circumference of the affect-ed and unaffected arm, and calculated according to the following formula: [(total circumference of the affected arm - total circumference of the unaffected arm) / - total circumference of the unaffected arm] x 100, where $0 \%$ indicates the same total circum-ferences of two arms. The degree of lymphoedema reduction was calculated by the following formu-la: (total circumference of the affected arm before treatment - total circumference of the affected arm after treatment/ total circumference of the affected arm before treatment - total circumference of the unaffected arm) x $100 .{ }^{14-16}$

\section{Bandage compliance}

All patients received instructions for wearing the bandages as long as possible ie, until the next treat-ment day. The bandage-carrying compliance was evaluated through the so-called "bandage log" in which all patients registered the exact time of ap-plication and removal of bandages daily, based on which the total number of hours under the ban-dage was calculated. 


\section{Statistics}

Descriptive statistics methods were used to describe all data in the study. Numerical data asso-ciated with the percentage of lymphoedema re-duction were identified by Pearson correlation. Categorical data were analysed by the indepen-dent samples t-test. Factors with $p<0.05$ in the stated analyses were selected as final predictors for multivariate linear regression analysis. All analyses were carried out using SPSS Version 21.0 for Windows. The result was significant if the p-value was less than 0.05 .

\section{Results}

The prospective study included 51 female patients with secondary arm lymphoedema after breast cancer treatment. Mean age was $58.1 \pm$ 8.0 (95 \% CI: 55.8 - 60.3), median of BMI was 28.4 $\mathrm{kg} / \mathrm{m}^{2}$ (95 \% CI: 27.2 - 29.6). The largest number of patients (47.1\%) were in the category of overweight, $29.4 \%$ were obese patients, $21.5 \%$ were in normal range and only 1 patient (2.0\%) was

\section{Table 1: Patients' characteristics}

\begin{tabular}{|c|c|c|c|}
\hline Characteristics & N (\%) & Mean \pm SD & $\begin{array}{c}\text { Range } \\
\text { (min-max) }\end{array}$ \\
\hline Patients' age (years) & & $58.1 \pm 8.0$ & $41.0-77.0$ \\
\hline Time from surgery (months) & & $53.4 \pm 50.0$ & $3.0-185.0$ \\
\hline \multicolumn{4}{|l|}{ Type of breast surgery } \\
\hline Rradical mastectomy & $30(58.8)$ & & \\
\hline Breast conserving surgery & $21(41.2)$ & & \\
\hline Number of lymph nodes removed & & $14.2 \pm 6.6$ & $3.0-42.0$ \\
\hline Number of lymph nodes involved & & $2.8 \pm 6.1$ & $0.0-32.0$ \\
\hline \multicolumn{4}{|l|}{ Therapy } \\
\hline Chemotherapy & $37(72.5)$ & & \\
\hline Radiotherapy & $38(74.5)$ & & \\
\hline Hormonal & $37(72.5)$ & & \\
\hline Duration of lymphoedema (months) & & $36.5 \pm 43.9$ & $0.5-170.0$ \\
\hline Time until lymphoedema onset (months) & & $17.0 \pm 22.9$ & $0.0-124.0$ \\
\hline Size of lymphoedema before CDT (\%) & & $6.99 \pm 5.36$ & $1.99-25.0$ \\
\hline Degree of lymphoedema reduction (\%) & & $63.7 \pm 28.6$ & $13.8-100$ \\
\hline \multicolumn{4}{|l|}{ Reporting pain and other symptoms in the arm } \\
\hline Yes & $44(86.3)$ & & \\
\hline No & $7(13.7)$ & & \\
\hline \multicolumn{4}{|l|}{ Hystory of erysipelas } \\
\hline Yes & $7(13.7)$ & & \\
\hline No & $44(86.3)$ & & \\
\hline Body mass index - BMI $\left(\mathrm{kg} / \mathrm{m}^{2}\right)$ & & $28.4 \pm 4.3$ & $17.9-37.9$ \\
\hline Underweight $(<18.5)$ & $1(2.0)$ & & \\
\hline Normal range (18.50-24.99) & $11(21.5)$ & & \\
\hline Underweight (25.00-29.99) & $24(47.1)$ & & \\
\hline Obese $(>30)$ & $15(29.4)$ & & \\
\hline \multicolumn{4}{|l|}{ Comorbidity (medication for) } \\
\hline Hypertension & $23(45.1)$ & & \\
\hline Heart disease & $9(17.6)$ & & \\
\hline Thyroid problems & $12(23.5)$ & & \\
\hline Diabetes & $4(7.8)$ & & \\
\hline Venous insufficiency in the lower limbs & $5(9.8)$ & & \\
\hline Osteoporosis & $8(15.7)$ & & \\
\hline Others & $5(9.8)$ & & \\
\hline
\end{tabular}

in the category of underweight. The average dura-tion of lymphoedema was $36.5 \pm 43.9$ months (95 \% CI: 24.1 - 48.8). The mean degree of lymphoedema reduction was $63.7 \pm 28.6 \%$. The mean compliance to bandages was $217.5 \pm 97.8$ hours (95 \% CI: 190.0 - 245.0) and 7 (13.7 \%) patients had a history of erysipelas of the ipsilat-eral arm. Table 1 shows characteristics of the pa-tients.

The size of lymphoedema before therapy was sta-tistically significantly negatively correlated with degree of lymphoedema reduction $(\mathrm{p}<$ 0.001). Also, there was statistically significant negative correlation between patients' age and percentage of lymphoedema reduction $(\mathrm{p}<0.05)$. The degree of lymphoedema reduction in patients with his-tory of erysipelas, was statistically significantly lower than in those who did not have erysipelas $(\mathrm{p}<0.01)$ (Table 2$)$.

\section{Table 2: Factors accociated with efficacy of CDT}

\begin{tabular}{|c|c|c|c|c|}
\hline \multirow{2}{*}{ Variable } & \multicolumn{2}{|c|}{$\begin{array}{l}\text { Pearson } \\
\text { correlation }\end{array}$} & \multicolumn{2}{|c|}{$\begin{array}{c}\text { Independent samples } \\
\text { T-test }\end{array}$} \\
\hline & $\mathbf{R}$ & $\mathbf{p}$ & $\mathbf{T}$ & p \\
\hline Patients' age (years) & -0.280 & 0.047 & & \\
\hline Time from surgery (months) & -0.129 & 0.367 & & \\
\hline \multicolumn{5}{|l|}{ Type of breast surgery } \\
\hline Number of lymph nodes removed & -0.186 & 0.191 & -0.608 & 0.546 \\
\hline Number of lymph nodes involved & -0.016 & 0.914 & & \\
\hline \multicolumn{5}{|l|}{ Chemotherapy } \\
\hline Radiotherapy & & & -0.508 & 0.614 \\
\hline Hormonal therapy & & & 0.109 & 0.914 \\
\hline Duration of lymphoedema (months) & -0.082 & 0.569 & 0.142 & 0.888 \\
\hline $\begin{array}{l}\text { Time until lymphoedema onset } \\
\text { (months) }\end{array}$ & -0.124 & 0.386 & & \\
\hline Size of lymphoedema before CDT & -0.710 & 0.000 & & \\
\hline $\begin{array}{l}\text { Reporting pain and other symptoms } \\
\text { in the arm }\end{array}$ & & & -0.406 & 0.687 \\
\hline Hystory of erysipelas & -0.129 & 0.367 & -3.808 & 0.002 \\
\hline \multicolumn{5}{|l|}{ Body mass index - BMI $\left(\mathrm{kg} / \mathrm{m}^{2}\right)$} \\
\hline Comorbidity (yes/no) & & & 0.516 & 0.608 \\
\hline Number of comorbidities & 0.076 & 0.598 & & \\
\hline Compliance to bandages (hours) & 0.008 & 0.956 & & \\
\hline
\end{tabular}


Table 3: Predictors of complete decongestive treatment (CDT) efficacy after multivariate analysis

\begin{tabular}{lccc}
\hline Factors & $\begin{array}{c}\beta \text { coefficient of } \\
\text { linear regression }\end{array}$ & t-value & $\mathbf{p}$ \\
\hline Size of lymphoedema before the CDT & -0.553 & -6.207 & 0.000 \\
\hline Hystory of erysipelas & 0.273 & 3.078 & 0.004 \\
\hline Patients' age (years) & -0.200 & -2.354 & 0.023 \\
\hline
\end{tabular}

Influence of certain predictors on the degree of lymphoedema reduction was evaluated by multi-variate linear regression analysis. The results showed that the model explained $65.4 \%$ of the total variance, $(F=24.579, p=0.000)$. When ob-serving each individual predictor, statistically most significant contribution showed the size of lymphoedema before the therapy ( $p<0.001)$, then history of erysipelas $(p<0.01)$, and patients' age $(\mathrm{p}<0.05)$. Table 3 shows predictors of CDT efficacy after multivariate analysis with the per-centage of lymphoedema reduction as dependent variable.

\section{Discussion}

In this study, the influence of independent predictors on the success of decongestive therapy of reast cancer-related arm lymphoedema was investigated. Younger age and lower size of lymphoedema before CDT were identified as predictors associated with better response to treatment. The history of erysipelas was associated with a poor outcome of CDT.

The most important predictor of the degree of reduction of lymphoedema was the size of lymphoedema before the therapy: the lower the size of lymphoedema before the treatment was, the greater the degree of reduction achieved. Efficacy of CDT is better, if the therapy starts as earlier as possible, when lymphoedema is less pronounced. Similar results were obtained by other authors. $^{14,15,17}$

The younger age was also a predictor of better therapy response. Lia SF et al also reported that younger age would predict CDT efficacy, believing that older patients have poor compliance with bandages.14 That could not be concluded in the present study. In the study of predictors of lower limb lymphoedema, Vignes et al reported that older patients had better treatment outcomes. The average age of patients in this study (45.8; range 32-60.4) was considerably less than in mentioned study (58.1; range 41-77). ${ }^{18}$
The most common lymphoedema complication is erysipelas. It is an infection that involves the superficial layer of the skin with primarily affectsthe lymphatic vessels (lymphangitis). Upper limb erysipelas occurs in up to $24 \%$ of women aftersurgical treatment for breast cancer followinglymphatic system damage. Lymphoedema is considerable risk factor for reappearance of erysipelas. ${ }^{19-21}$

The only study that identified previous erysipelas as predictor of the efficacy of CDT was a study of primary lower extremity lymphoedema. But, in that study patients with previous episode(s) of erysipelas obtained higher lymphoedema volume reduction.18 History of earlier erysipelas has proved to be an individually negative significant predictor in the present research. Considering total of patients who had erysipelas in this study was 7 (13.7\%), this clinical feature requires further research.

Erysipelas may have negative impact on CDT efficacy. Every episode od erysipelas affects the lymphatic vessels, aggravating of pre-existing lymphatic impairment and worsening lymphoedema.

A surprising finding in these results is that bandage-carrying compliance was not associated with better treatment response. Bandage compliance is generally considered a factor influencingCDT outcomes. According to the findings of some authors, bandage-carrying compliance is a dominant predictive factor of CDT effectiveness, not only after the intensive phase of treatment, but also during the maintenance phase. ${ }^{15,22}$

Forner-Cordero et al concluded that good bandage

compliance improved the percent reduction of lymphoedema by $25 \%$ compared with fair or bad bandage compliance in breast cancer-linked lymphoedema. ${ }^{15}$

The present study is, to authors' knowledge, the only study that evaluated bandage-carrying compliance using a "bandage log" and compared the total number of hours of wearing a bandage with the degree of lymphoedema reduction. The average number of hours of bandage wearing in this study was $217.5 \pm 97.8$ hours (range $81-471$ ). If the criteria from the Forner-Cordero' study were used, $72.5 \%$ of patients in this study would have bad bandage-carrying compliance, $25.5 \%$ fair compliance, and only 1 patient ( $2 \%$ ) good bandage-carrying compliance. Since most patients 
had poor bandage-carrying compliance, this factor requires further examination in a larger number of patients.

BMI and duration of lymphoedema also were not associated with better response to CDT. The results of other studies are contradictory. Vignes et al demonstrated that duration of lymphoedema and BMI were correlated with a greater absolute reduction, but not a relative decrease in lymphoedema volume. ${ }^{23}$ Forner-Cordero at al found that the duration of lymphoedema does not affect the outcome of the therapy and that patients may benefit from treatment long time after symptoms appear. ${ }^{15}$

The authors consider that the most important factor in keeping lymphoedema under control is the

regular administration of CDT to reduce swelling and that the largest lymphoedemas are not the oldest.

It is well known that overweight or obesity, expressed as a BMI greater than 25.0 and 30.0 respectively, is important risk factor for secondary lymphoedema.24-26 The effect of BMI on CDT outcome has been described in some studies, ${ }^{18,22}$, ${ }^{23}$ but in this one, such a result was not obtained. The

reason could be that most of the patients in this study were in the pre-obese or obese category, and only $21.5 \%$ were in the normal range.

The breast cancer treatment characteristics (time from surgery, type of breast surgery, number of lymph nodes removed and involved, treatment before and/or after surgery) did not affect CDT efficacy in present study.

\section{Study strengths and limitations}

The present study was conducted at an institution specialised in the treatment of patients with lymphoedema and only included patients with arm lymphoedema linked to breast cancer. The study was prospective and monocentric. All patients received homogenous CDT protocol and MLD was carried out by two trained physiotherapists and under the supervision of the researcher. The main restriction of this study is little sample size. Forthcoming research with greater number of patients is necessary.

\section{Conclusion}

Size of lymphoedema before treatment is the most crucial prognostic factor of the efficacy of CDT in the patients with breast cancer-linked arm lymphoedema. The present study also identified history of erysipelas and patients age as independent predictors of the CDT efficacy.

Although this study did not show statistical significance for the bandage-carrying compliance, BMI and duration of lymphoedema, these factors should be paid attention in the further prospective studies with a larger number of patients.

\section{Acknowledgements}

None.

\section{Conflict of interest}

None.

\section{References}

1. Bains SK, Peters AM, Zammit C, Ryan N, Ballinger J, Glass DM et al. Global abnormalities in lymphatic function following systemic therapy in patients with breast cancer. Br J Surg. 2015; 102(5):534-40.

2. Cariati M, Bains SK, Grootendorst MR, Suyoi A, Peters $\mathrm{AM}$, Mortimer P et al. Adjuvant taxanes and the development of breast cancer-related arm lymphoedema. $\mathrm{Br}$ J Surg 2015; 102(9):1071-8.

3. Lopez Penha TR, van Roozendaal LM, Smidt ML, Boersma LJ, von Meyenfeldt MF, Voogd ACet al. The changing role of axillary treatment in breast cancer: Who will remain at risk for developing arm morbidity in the future? Breast. 2015; 24(5):543-7.

4. Warren LE, Miller CL, Horick N, Skolny MN, Jammallo LS, Sadek BT, et al. The impact of radiation therapy on the risk of lymphedema after treatment for breast cancer: a prospective cohort study. Int J Radiat Oncol Biol Phys. 2014; 88(3):565-71.

5. Sakorafas GH, Peros G, Cataliotti L, Vlastos G. Lymphedema following axillary lymph node dissection for breast cancer. Surg Oncol. 2006; 15(3): 153-65.

6. Armer JM, Hulett JM, Bernas M, Ostby P, Stewart BR, Cormier JN. Best-Practice Guidelines in Assessment, Risk Reduction, Management, and Surveillance for Post-Breast Cancer Lymphedema. Curr Breast Cancer Rep 2013;5(2):134-44. 
7. Cheifetz O, Haley L. Management of secondary lymphedema related to breast cancer. Can Fam Physician. 2010; 56 (12): 1277-84.

8. Jeffs E, Ream E, Taylor C, Bick D. Clinical effectiveness of decongestive treatments on excess arm volume and patient-centered outcomes in women with early breast cancer-related arm lymphedema: a systematic review. JBI Database System Rev Implement Rep. 2018 Feb; 16(2): 453-506.

9. Borman P. Lymphedema diagnosis, treatment, and follow-up from the viewpoint of physical medicine and rehabilitation specialists. Turk J Phys Med Rehabil. 2018 Sep; 64(3): 179-97.

10. Yamamoto R, Yamamoto T. Effectiveness of the treatment-phase of two-phase complex decongestive physiotherapy for the treatment of extremity lymphedema. Int J Clin Oncol. 2007; 12(6):463-8.

11. Koul R, Dufan T, Russell C, Guenther W, Nugent Z, Sun $\mathrm{X}$ et al. Efficacy of complete decongestive therapy and manual lymphatic drainage on treatment-related lymfodema in breast cancer. Int J Radiat Oncol Biol Phys. 2007; 67(3):841-6.

12. Karadibak D, Yavuzsen T, Saydam S. Prospective trial of intensive decongestive physiotherapy for upper extremity lymphedema. J Surg Oncol. 2008; 97(7):572-7.

13. Buragadda S, Alhusaini AA, Melam GR, Arora N. Effect of complete decongestive therapy and a home program for patients with post mastectomy lymphedema. J Phys Ther Sci. 2015; 27(9):2743-8.

14. Liao SF, Li SH, Huang HY, Chen ST, Kuo SJ, Chen DR, Wei TS. The efficacy of complex decongestive physiotherapy (CDP) and predictive factors of lymphedema severity and response to CDP in breast cancer-related lymphedema (BCRL). Breast 2013 Oct; 22(5):703-6.

15. Forner-Cordero I, Muñoz-Langa J, Forner-Cordero A, DeMiguel-Jimeno JM. Predictive factors of response to decongestive therapy in patients with breast-cancer-related lymphedema. Ann Surg Oncol. 2010 Mar; 17(3): 744-51.

16. Hamner JB, Fleming MD. Lymphedema therapy reduces the volume of edema and pain in patients with breast cancer. Ann Surg Oncol. 2007; 14(6):1904-8.

17. Liao SF, Li SH, Huang HY. The efficacy of complex decongestive physiotherapy (CDP) and predictive factors of response to CDP in lower limb lymphedema (LLL) after pelvic cancer treatment. Gynecol Oncol. 2012 Jun; 125(3): 712-5.

18. Vignes S, Simon L, Benoughidane B, Simon M, Fourgeaud C. Clinical and Scintigraphic Predictors of Primary Lower Limb Lymphedema-Volume Reduction During Complete Decongestive Physical Therapy. Phys Ther 2020 May 18; 100(5): 766-72.

19. Dalal A, Eskin-Schwartz M, Mimouni D, Ray S, Days W, Hodak et al. Interventions for the prevention of recurrent erysipelas and cellulitis. Cochrane Database Syst Rev 2017 Jun 20; 6(6):CD009758.

20. Jendoubi F, Rohde M, Prinz JC. Intracellular Streptococcal Uptake and Persistence: A Potential Cause of Erysipelas Recurrence. Front Med (Lausanne) 2019 Jan 29; 6:6.

21. Inghammar M, Rasmussen M, Linder A. Recurrent erysipelas - risk factors and clinical presentation. BMC Infect Dis. 2014; 14: 270.

22. Vignes S, Porcher, Arrault M, Dupuy A. Factors influencing breast cancer-related lymphedema volume after intensive decongestive physiotherapy. Support Care Cancer. 2011 Jul; 19(7): 935-40.

23. Vignes S, Porcher R, Champagne A, Dupuy A. Predictive factors of response to intensive decongestive physiotherapy in upper limb lymphedema after breast cancer treatment: a cohort study. Breast cancer Res Treat. 2006 Jul; 98(1): 1-6.

24. Lopez Penha TR, van Roozendaal LM, Smidt ML, Boersma LJ, von Meyenfeldt MF, Voogd ACet al. The changing role of axillary treatment in breast cancer: Who will remain at risk for developing arm morbidity in the future? Breast. 2015; 24(5):543-7.-BMI

25. Basta MN, Fischer JP, Kanchwala SK, Silvestre J, Wu LC, Serletti JM et al. A Propensity-Matched Analysis of the Influence of Breast Reconstruction on Subsequent Development of Lymphedema. Plast Reconstr Surg. 2015; 136(2):134- 43.

26. Soran A, Menekse E, Girgis M, DeGore L, Johnson R. Breast cancer-related lymphedema after axillary lymph node dissection: does early postoperative prediction model work? Support Care Cancer. 2016;24(3):1413-9. 